\title{
PROPROTEIN CONVERTASE SUBTILISIN/KEXIN TYPE 9 (PCSK9), SOLUBLE LECTIN-LIKE OXIDIZED LDL RECEPTOR 1 (sLOX-1) AND ANKLE BRACHIAL INDEX IN SHORT TERM OVERT HYPOTHYROIDISM
}

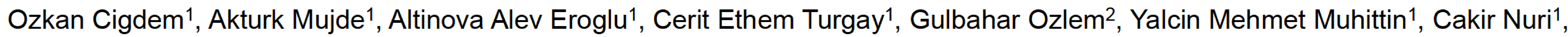 \\ Toruner Fusun Balos ${ }^{1}$ \\ ${ }^{1}$ Gazi University Faculty of Medicine, Department of Endocrinology and Metabolism, Ankara, Turkey \\ ${ }^{2}$ Gazi University Faculty of Medicine, Department of Biochemistry, Ankara, Turkey
}

\section{Objectives:}

\section{Methods:}

\section{Results:}

Differentiated thyroid cancer is a common endocrine cancer and its incidence increases worldwide [1]. Radioactive iodine (RAl) therapy is a treatment option for patients with differentiated thyroid cancer [2]. Serum thyroid stimulating hormone $(\mathrm{TSH})$ can be raised endogenously by thyroid hormone withdrawal (THW) or exogenously by administration of recombinant human TSH for RAl ablation therapy or whole body RAI scan [3]. THW results in rapid and severe short term hypothyroidism which may lead to undesirable clinical consequences. Short term rapid overt hypothyroidism may lead to changes in cardiovascular system [4]. In this study, we aimed to evaluate serum PCSK9, sLOX-1, lipid levels, $A B I$, and their relationships with each other in short term overt hypothyrodism due to THW in patients with thyroid cancer who undergo RAI remnant ablation or RAI scan.

Twenty-one patients (20 female, 1 male) who require RAI ablation or RAl scan and thirty-six ( 35 female, 1 male) age, sex, body mass index (BMI) similar euthyroid healthy control subjects were enrolled into this prospective study. In all patients who require RAl ablation or RAI scan, serum TSH was raised endogenously due to THW. We evaluated patients both in subclinical thyrotoxic period when they were on suppressive levothyroxine therapy and overt hypothyroid period due to THW for four weeks. Anthropometric parameters were obtained from all patients and healthy control subjects. ABI measurements were performed. Thyroid function tests, fasting plasma glucose, lipid parameters, PCSK-9 and sLOX-1 levels were evaluated from serum samples.

Total cholesterol, LDL cholesterol, triglycerides and Apo B levels were increased in short term overt hypothyroidism compared to control group $(p<0.001)$. Patients with short term overt hypothyroidism had significantly increased PCSK9 levels compared to control group $(p<0.001)$. PCSK9 levels were also found to be increased before THW when compared to control group ( $p=0.004)$. sLOX-1 levels were not different between patients with short term overt hypothyroidism and control group $(p=0.27)$. ABI was found to be significantly decreased in patients with short term overt hypothyroidism compared to control group $(p=0.02)$. PCSK9 levels were correlated negatively with $A B I(r=-0.38, p=0.004)$.

\begin{tabular}{|c|c|c|c|c|c|c|}
\hline & 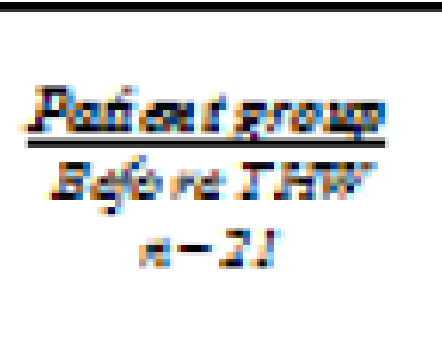 & 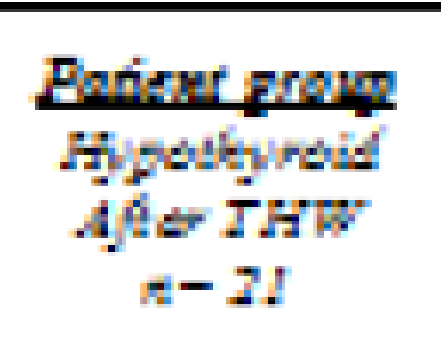 & 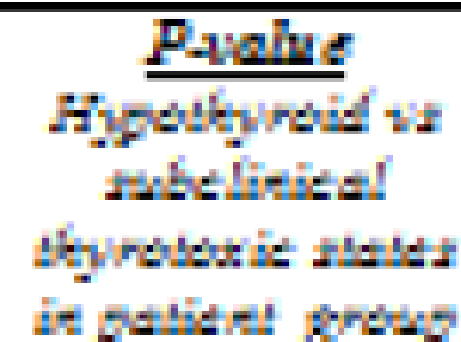 & $\underset{n=-15}{C O \text { ntrols }}$ & 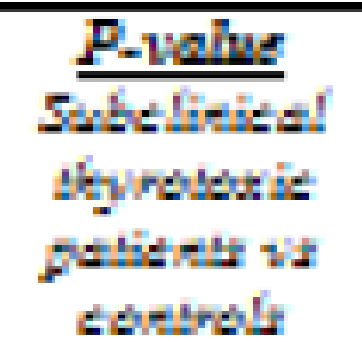 & 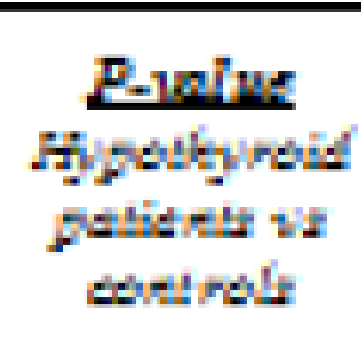 \\
\hline Age (6ses) & $43.91=10.44$ & & & $41.78=5.94$ & 0.31 & \\
\hline Gevd er (TM) & 2011 & & & $35 / 1$ & 0.75 & \\
\hline $\mathrm{BMI}\left(\mathrm{gz} \mathrm{m}^{2}\right)$ & $28.39=4.90$ & $29.35=4.99$ & -0.001 & $26.37=4.65$ & 0.13 & 0.02 \\
\hline TSH $(\mu \pi / m)$ & $0.14(1.07)$ & $1000(57.45)$ & -0.001 & $211=104$ & $\$ 0.001$ & $\$ 0.001$ \\
\hline FT4 (ng dif) & $1.44=0.28$ & $0.40(0.03)$ & $=0.001$ & $1.09=0.16$ & $\$ 0.01$ & -4001 \\
\hline FT3 $(\rho \mathrm{g}: \mathrm{m} 1)$ & $3.07=1.10$ & $1.28=0.48$ & $-\infty 0.001$ & $291=0.26$ & 0.02 & $\$ 0.001$ \\
\hline FBG (ng dif) & $9277=10.97$ & $\operatorname{ss} .22=13.77$ & 0.10 & $900(7.75)$ & 0.58 & 0.15 \\
\hline T.chd deterol (mg di) & $211.35=36.77$ & $276.0(500)$ & -0.001 & $201.09=34.83$ & 0.27 & $\$ 0.001$ \\
\hline $\operatorname{LDL}, \mathcal{C}(\mathrm{mg} \in \mathbb{L})$ & $134.39=31.24$ & $20056=53.30$ & $=0.001$ & $127.35=18.79$ & 0.39 & $\$ 0.001$ \\
\hline HDL $-C(\operatorname{mg}$ al) & $50.57=11.24$ & $18.50=13.21$ & $=0.001$ & $53.36=1095$ & 0.37 & 0.09 \\
\hline $\mathrm{TG}(\mathrm{mg}$ dil) & $108.0(73.0)$ & $1520(5250)$ & 0.15 & $94.50058 .50)$ & 0.10 & $\$ 0.001$ \\
\hline Apo AI (mg di) & $1420(37.50)$ & $15690=25.47$ & 002 & $149.12=20.55$ & 0.75 & 0.22 \\
\hline Apo B (mg di) & $101.0=21.81$ & $140.04=30.34$ & $=0.001$ & $9047=19.01$ & 0.06 & $\$ 0.001$ \\
\hline Lipop rotais-a (mg di) & $20.60=172$ & $19.45(18.77)$ & 0.56 & $17.75(16.82)$ & 0.23 & 0.65 \\
\hline PCSKO (agmi) & $287.98(87.29)$ & $317.57(9204)$ & 021 & $209.46(97.03)$ & 0.004 & -4.001 \\
\hline$=\mathrm{LOX}-1(\rho \mathrm{gI})$ & $95.83=42.98$ & $128.50=78.56$ & 0.06 & 81.21(95.05) & 0.54 & 0.27 \\
\hline ABI & $1.13=0.12$ & $1.0(0.29)$ & 0.06 & $120020)$ & 0.46 & 0.02 \\
\hline
\end{tabular}

To the best of our knowledge, our study is the first study in the literature investigating prospectively PCSK 9 and sLOX-1 levels and their association with $A B I$ as cardiovascular risk parameter in short term overt hypothyroidism. The results of our study support the hypothesis that short term overt hypothyroidism leads to increased PCSK9 levels, lipid changes as well as decreased $A B I$ which is an indicator of atherosclerosis in short term overt hypothyroidism due to THW. Therefore, the possible cardiovascular consequences of short term overt hypothyroidism should be considered especially in patients with increased cardiovascular risk. 